\title{
Response of the warm absorber cloud to a variable nuclear flux in active galactic nuclei
}

\author{
L. Chevallier ${ }^{1,2}$, B. Czerny ${ }^{2}$, A. Różańska ${ }^{2}$, and A. C. Gonçalves ${ }^{1,3}$ \\ 1 Observatoire de Paris-Meudon, LUTH, Meudon, France \\ e-mail: loic.chevallier@obspm.fr \\ 2 Copernicus Astronomical Center, Bartycka 18, 00-716 Warsaw, Poland \\ 3 Centro de Astronomia e Astrofísica da Universidade de Lisboa, Observatório Astronómico de Lisboa, Tapada da Ajuda, \\ 1349-018 Lisboa, Portugal
}

Received 22 May 2006 / Accepted 20 December 2006

\section{ABSTRACT}

\begin{abstract}
Recent modeling of the warm absorber in active galactic nuclei has proved the usefulness of constant total (gas plus radiation) pressure models, which are highly stratified in temperature and density. We explore the consistency of those models when the typical variation of the flux from the central source is taken into account. We have performed a variability study of the warm absorber response, based on timescales and our photoionization code TITAN. We show that the ionization and recombination timescales are much shorter than the dynamical timescale. Clouds very close to the central black hole will maintain their equilibrium since the characteristic variability timescales of the nuclear source are longer than cloud timescales. For more distant clouds, the density structure has no time to vary in response to the variations in the temperature or ionization structure, and such clouds will show the departure from the constant pressure equilibrium. We explore the impact of this departure on the observed properties of the transmitted spectrum and soft X-ray variability: (i) non uniform velocities, on the order of sound speed, appear due to pressure gradients, up to typical values of $100 \mathrm{~km} \mathrm{~s}^{-1}$. These velocities lead to the broadening of lines. This broadening is usually observed and very difficult to explain otherwise. (ii) Energy-dependent fractional variability amplitude in soft X-ray range has a broader hump around $\sim 1-2 \mathrm{keV}$, and (iv) the plot of the equivalent hydrogen column density vs. ionization parameter is steeper than for equilibrium clouds. The results have the character of a preliminary study and should be supplemented in the future with full time-dependent radiation transfer and dynamical computations.
\end{abstract}

Key words. radiative transfer - line: identification - galaxies: Seyfert - X-rays: galaxies - galaxies: active

\section{Introduction}

The environment of radio-quiet active galactic nuclei (AGN) is still poorly understood. The activity is powered by a black hole but the accretion flow is accompanied by an outflow of the material. The inflow is in the form of an accretion disk which, further out, has the form of a dusty/molecular torus, and the radial velocities of the inflow are not measured directly. The outflow takes place in a wide range of angles around the symmetry axis, but the geometry and the nature of this flow is unclear. The presence of the outflow manifests itself best as broad absorption lines (BAL) of the optical/UV spectra of some quasars, narrow absorption lines (NAL) in other quasars or in Seyfert galaxies, or as X-ray warm absorbers (WA) in Seyfert galaxies. These absorption features allow determination of the outflow velocity of the plasma along the line of sight, but any estimates of the spatial distribution of the plasma, or even of a representative distance of the absorber from the nucleus, are difficult.

One important question is whether this outflow is roughly a continuous wind with a shallow density gradient or a highly clumpy medium with very dense clouds embedded in lowdensity surrounding plasma. The model of a continuous wind was proposed by Murray \& Chiang (1995) and subsequently studied e.g. by Murray \& Chiang (1997, 1998), Proga et al. (2000, 2004), Pietrini \& Torricelli-Ciamponi (2000). On the other hand, the strong X-ray irradiation of the outflowing material may lead to thermal instability (Krolik et al. 1981), thus resulting in clumpiness in a part of the flow. Therefore, clumpy outflow is advocated by a number of authors (e.g. Smith \& Raine 1988; Netzer 1993; Krolik \& Kriss 1995; Netzer 1996). A narrow stream of clumpy outflow is advocated by Elvis (2000), while narrow continuous streamers are favored by Steenbrugge et al. (2005). Recently, an advanced model of the clumpy outflow has been developed by Chelouche \& Netzer (2005), based on previous extensive studies of the wind acceleration mechanisms.

The issue can in principle be solved directly on observational grounds. However, X-ray data even from grating instruments on board Chandra or XMM-Newton telescopes cannot resolve kinematical components narrower than $\sim 100 \mathrm{~km} \mathrm{~s}^{-1}$. High-resolution UV data show absorption lines with the kinematical widths $\sim 100 \mathrm{~km} \mathrm{~s}^{-1}$ (e.g., NGC 3783, Gabel et al. 2003) or $50-60 \sim 100 \mathrm{~km} \mathrm{~s}^{-1}$ (e.g., NGC 7469, Scott et al. 2005). These results are consistent with X-ray data, but it is still not clear whether we probe the same part of the flow with $\mathrm{X}$-ray and UV data. Spectral analysis of the X-ray data suggests that one zone model does not represent the observations well and at least two photoionization regions are required to explain the presence of lines from the gas in different ionization states (NGC 4051, Collinge et al. 2001; NGC 5548, Kaastra et al. 2002; NGC 3783, Kaspi et al. 2002; Netzer et al. 2003 and Krongold et al. 2003; Mkn 304, Piconcelli et al. 2004; H0557-385, Ashton et al. 2006). 
On the theoretical side, it is not clear whether it is even possible to achieve and sustain strong clumpiness of the medium. The time evolution of the warm absorber medium has not been studied in great detail so far. Additionally, AGN have been known for their variability in the X-ray band since the EXOSAT data. Variable irradiation changes the conditions within the cloud and might even destroy the cloud. Also small compact cloud will respond to variable X-ray flux in a different way than an extended medium.

In the present paper we concentrate on a single warm absorber cloud under constant pressure. We consider a cloud of considerably high column density and non-negligible optical depth. Such a cloud is strongly stratified since the density profile is obtained self-consistently from the condition of total pressure equilibrium (see Dumont et al. 2002; Chevallier et al. 2006; Różańska et al. 2006), inducing a few sharp temperature drops due to thermal instabilities. Magnetic and turbulent pressure effects have been neglected in this study. It is well known that magnetic pressure may remove the thermal instabilities (Bottorff et al. 2000), but our cloud, if large enough, will still remain highly stratified with a shallower temperature and density gradient near the location of the previous thermal instability zone. It is the same with turbulent pressure.

The bright irradiated side of the cloud has a low density and is highly ionized but the density strongly increases inward (by a few orders of magnitude) and the medium becomes less ionized as we approach the dark side of the cloud. This medium has been used to fit the WA of NGC 3783 (Gonçalves et al. 2006). Here we study the response of the cloud to the varying X-ray flux, with the aim of testing the possibility of strong clumpiness in the warm absorber. Using the time-independent code TITAN, we estimate the evolution of our cloud at the basis of timescales, determined separately in different parts of our highly stratified cloud. The cloud, initially in total (gaseous + radiative) pressure equilibrium, is disturbed; pressure is no longer constant and various parts of the cloud expand/contract. We estimate the observational consequences of the cloud departure from equilibrium. Finally we show how the behavior of our cloud, whose distance from the central source is unknown, varies with this distance.

In the next section, we recall some generalities about the model and our photoionization code, and we give formulae for timescales that are explained in the Appendix. Results are presented in Sect. 3. Section 4 is dedicated to the discussion of the physical and observational implications of these models.

\section{Model}

We study the response of a single warm absorber cloud to a change in the incident X-ray radiation flux, $F$. Using a stationary radiative transfer code, we determine the kinds of equilibrium solutions:

$$
\begin{aligned}
& \text { - total pressure and thermal equilibrium; } \\
& \text { - only thermal equilibrium. }
\end{aligned}
$$

The first type of solution is a cloud in total pressure equilibrium. It corresponds to a cloud that is in complete equilibrium with the incident flux. The second type of solution is obtained by allowing for a change in the irradiating flux but assuming that the timescale of this change, $t_{\mathrm{X}}$, is shorter than the timescale needed to restore the pressure equilibrium. In this case we perform the computations of the new thermal and ionization equilibrium, but we retain the original density profile. This second solution will describe the cloud out of pressure equilibrium. The applicability of either the first or the second solution depends on the cloud location and the variability properties of the nucleus.

The radiative transfer used in both cases is the code TITAN (Dumont et al. 2000, 2003). This 1D code works in planeparallel symmetry but uses the accelerated lambda iteration (ALI) method to provide fully-consistent multi-angle radiative transfer computations for both continuum and lines (Rybicki \& Hummer 1991, and references therein). Using an accurate radiative transfer method is important in such a problem, where radiation pressure variation within the cloud plays an important role, especially in determining the location of temperature drops due to thermal instabilities. We consider about 1000 spectral lines, mainly in the X-ray range of the 10 most abundant species in the universe, i.e. $\mathrm{H}, \mathrm{He}, \mathrm{C}, \mathrm{N}, \mathrm{O}, \mathrm{Ne}, \mathrm{Mg}, \mathrm{Si}, \mathrm{S}$, and Fe. Throughout the paper we use cosmic abundances (Allen 1973), which are constant in the cloud.

The complete equilibrium state is characterized by the local density at the irradiated face of the cloud $n_{\mathrm{o}}$, the hydrogen column density of the cloud $N_{\mathrm{H}}$, and the properties of the incident radiation (a power-law of index 1 from $10 \mathrm{eV}$ to $100 \mathrm{keV}$, and the ionization parameter $\xi=L / n_{\mathrm{o}} R^{2}$, where $L$ is the bolometric luminosity of the source, $R$ the distance of the medium from the source). The density profile is determined consistently from the condition of the total pressure equilibrium. The off-equilibrium state is characterized by a new value of the ionization parameter $\xi$, but the density profile is taken from the previous equilibrium solution.

We first analyze the timescales for various processes within the cloud in full equilibrium, in order to establish selfconsistency of the cloud response. The light travel time is given by

$t_{\text {light }}=\frac{H}{c}$,

where $H$ is the geometrical size of a whole cloud or of a zone under consideration. In our case this timescale is relevant since the cloud is optically thin to the continuum. This low (but not negligible) optical depth is caused by significant ionization of the medium even on the dark side of the cloud.

If the irradiation changes, the whole volume of the cloud responds to the decrease/increase in photon flux. Therefore, we generally do not have well-defined ionization fronts here, as in the case of an expanding HII region around a star (e.g. Osterbrock 1974). The relevant description is provided, instead, by the ionization and recombination timescales. The ionization $t_{\text {ion }}$ and recombination $t_{\text {rec }}$ timescale for each ion is a local quantity within a cloud. However, we can introduce timescales for specific zones, or for a whole cloud, by analyzing which ions dominate in the various zones and which transitions are close to equilibrium (see the Appendix). We obtain the following estimates

$t_{\text {ion }}=6 \times 10^{-3} F_{16}^{-1}[\mathrm{~s}]$

and

$t_{\mathrm{rec}}=0.2 n_{12}^{-1}[\mathrm{~s}]$

where $F_{16}$ is the incident bolometric flux measured in units of $10^{16} \mathrm{erg} \mathrm{s}^{-1} \mathrm{~cm}^{-2}$, and $n_{12}$ the cloud hydrogen density at the surface in units of $10^{12} \mathrm{~cm}^{-3}$.

The local thermal timescale is defined as

$t_{\text {th }}=\frac{k T}{n_{\mathrm{e}} \Lambda}=12 T_{6} n_{12}^{-1} \Lambda_{23}^{-1}[\mathrm{~s}]$, 
where $k$ is the Boltzmann constant, $\Lambda_{23}$ the cooling function in units of $10^{-23} \mathrm{erg} \mathrm{cm}^{3} \mathrm{~s}^{-1}, T_{6}$ the temperature in units of $10^{6} \mathrm{~K}$, and the electron density $n_{\mathrm{e}} \sim 1.18 n_{\mathrm{H}}$, where $n_{\mathrm{H}}$ is the hydrogen density. The isothermal sound speed velocity is locally calculated as

$c_{\mathrm{s}}=\sqrt{\frac{2 k T}{m_{\mathrm{H}}}}=130 T_{6}^{1 / 2}\left[\mathrm{~km} \mathrm{~s}^{-1}\right]$

where $m_{\mathrm{H}}$ is the hydrogen atomic mass and the factor 2 accounts for mean molecular weight in an ionized medium. Equation (5) is relevant when taking only the gaseous pressure for a perfect gas into account. With such a stratified cloud, the dynamical timescale (i.e. time for restoration of pressure equilibrium) of a cloud zone of geometrical size $\Delta z=z_{2}-z_{1}$ is obtained through integration over $z$

$t_{\mathrm{dyn}}=\int_{z_{1}}^{z_{2}} \frac{\mathrm{d} z}{c_{\mathrm{s}}(z)}$

We generalize the results for a single cloud to a whole family of clouds at various distances from the nucleus. The initial configuration (i.e. the variation of all quantities in optical depth scale) practically does not depend on $n_{\mathrm{o}}$ (with values between $10^{5}$ to $10^{12} \mathrm{~cm}^{-3}$; see Różańska et al. 2006) for a given $\xi$ and $N_{\mathrm{H}}$. Since a change in the local density at the illuminated surface of the cloud is equivalent to a change in distance $R$ from the nucleus, we can take our cloud as representative of a whole family of self-similar clouds at various distances, so our model can be applied to several distances $R$, with appropriate dependence of $n_{\mathrm{o}}$ on radius $R$. We have $F(R) \propto R^{-2}$, the temperature profile only depends on $N_{\mathrm{H}}$ and $\xi$ and it remains the same whatever the value of $R$, and $n_{\mathrm{H}}$ scales with $n_{\mathrm{o}}$. We request $n_{\mathrm{o}}(R) \propto R^{-2}$, thus $H(R) \propto R^{2}$. Consequently all timescales defined in this section are proportional to $R^{2}$.

\section{Results}

\subsection{Initial state of the cloud}

We first model the cloud in complete total pressure equilibrium. For this reference cloud, we choose $\xi=10^{4} \mathrm{erg} \mathrm{cm} \mathrm{s}^{-1}$, $n_{\mathrm{o}}=10^{7} \mathrm{~cm}^{-3}$. We choose the largest $N_{\mathrm{H}} \sim 3 \times 10^{23} \mathrm{~cm}^{-2}$ allowed for the constant pressure cloud for such an incident spectrum (Chevallier et al. 2006). This choice for the column-density is consistent with computations of the cloud sizes by TorricelliCiamponi \& Courvoisier (1998), which take the thermal conduction into account. If we apply their expression for the cloud core scaling (Eq. (5)), taking their cloud dimensionless size $x=3 \times$ $10^{7}$ from their Figs. 4 and 5, and take the core temperature $3 \times$ $10^{4} \mathrm{~K}$ (i.e. the lowest temperature in our reference cloud), we obtain the expression for the column-density of the cloud $N_{\mathrm{H}} \sim 2 \times$ $10^{23} \mathrm{~cm}^{-2}$, independent of the core number density. The internal kinematics is set as random Gaussian movements with dispersion $100 \mathrm{~km} \mathrm{~s}^{-1}$, whose only effect is to increase the line width by Doppler effect.

The temperature profile across the cloud is shown in Fig. 1 as a function of the hydrogen column-density measured from the cloud surface. For such abundances, electron density is $n_{\mathrm{e}} \sim$ $1.18 n_{\mathrm{H}}$ and total density is $n \sim 2.265 n_{\mathrm{H}}$ ). The cloud develops four characteristic zones:

- low density high temperature extended zone (hot);

- intermediate zone (intermediate);

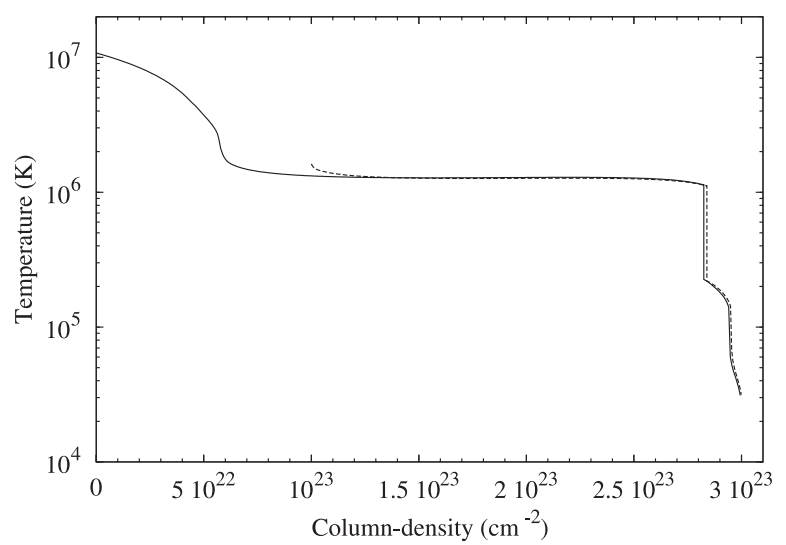

Fig. 1. The temperature profile of the cloud at the initial state $\xi=$ $10^{4} \mathrm{erg} \mathrm{cm} \mathrm{s}^{-1}$ (solid line). We also represent the case that is more typical of WA: $\log \xi=3$, for which maximal column-density is $2 \times$ $10^{23} \mathrm{~cm}^{-2}$ (dashed lines). This curve is shifted in column-density by a value $10^{23} \mathrm{~cm}^{-2}$ in order to compare with the $\log \xi=4$ case. Curves are not distinguishable.

Table 1. Characteristics of the four zones in this medium and for all the timescales considered in this study (see the text and Fig. 2). $t_{\text {light }}$ and $t_{\mathrm{dyn}}=\Delta z / c_{\mathrm{s}}$ are computed here zone by zone, and their maximal timescale is the sum over all the zones.

\begin{tabular}{ccccc}
\hline \hline & Hot & Intermediate & Outer & End \\
\hline$\Delta z[\mathrm{~cm}]$ & $3.4 \times 10^{15}$ & $1.6 \times 10^{15}$ & $8.4 \times 10^{12}$ & $9.9 \times 10^{11}$ \\
col. $\left[\mathrm{cm}^{-2}\right]$ & $5.8 \times 10^{22}$ & $2.3 \times 10^{23}$ & $1.3 \times 10^{22}$ & $5.3 \times 10^{21}$ \\
$T$ av. $[\mathrm{K}]$ & $8.0 \times 10^{6}$ & $1.3 \times 10^{6}$ & $1.9 \times 10^{5}$ & $4.8 \times 10^{4}$ \\
$n_{\mathrm{H}}$ av. $\left[\mathrm{cm}^{-3}\right]$ & $1.7 \times 10^{7}$ & $1.4 \times 10^{8}$ & $1.5 \times 10^{9}$ & $7.2 \times 10^{9}$ \\
$c_{\mathrm{s}}\left[\mathrm{km} \mathrm{s}^{-1}\right]$ & 370 & 150 & 57 & 28 \\
$t_{\text {light }}[\mathrm{s}]$ & $1.2 \times 10^{5}$ & $5.4 \times 10^{4}$ & 290 & 34 \\
$t_{\text {ion }}[\mathrm{s}]$ & $6 \times 10^{3}$ & $10^{3}$ & 100 & 30 \\
$t_{\text {rec }}[\mathrm{s}]$ & $2 \times 10^{4}$ & $2 \times 10^{3}$ & 100 & 20 \\
$t_{\text {th }}[\mathrm{s}]$ & $10^{7}$ & $2 \times 10^{5}$ & $10^{3}$ & 60 \\
$t_{\text {dyn }}[\mathrm{s}]$ & $9.3 \times 10^{7}$ & $1.1 \times 10^{8}$ & $1.5 \times 10^{6}$ & $3.5 \times 10^{5}$ \\
\hline
\end{tabular}

- high-density, low-temperature outer zone (outer);

- thin zone of highest density and lowest temperature (end).

The last zone is geometrically very thin, as can be seen in Table 1, but the hydrogen column density of this zone is significant because of the high density. For the considered cloud, the ratio of densities at the cold and hot surfaces is $\sim 10^{3}$, whatever the value of density $n_{\mathrm{o}}$ between $10^{5}$ and $10^{12} \mathrm{~cm}^{-3}$. The geometrical size of the reference cloud is $5 \times 10^{15} \mathrm{~cm}$, smaller by a factor of 6 than for a constant density cloud with the same column density and the same density at the cloud surface. The light travel time across the cloud is $1.7 \times 10^{5} \mathrm{~s}$. The typical value of $\xi$ for warm absorbers, estimated from the observational data, is instead $\sim 1000$ or lower, and these values are met in the interior of our reference cloud (i.e. intermediate zone, see Table 1) where the density is roughly ten times the density at the surface of our medium. Figure 1 confirms that models with $\log \xi=3$ and 4 behave similarly. For our $\log \xi=3$ model, $N_{\mathrm{H}} \sim 2 \times$ $10^{23} \mathrm{~cm}^{-2}$, which is now the maximum column density. In order to compare these temperature profiles, we have shifted the solution for $\log \xi=3$ by $10^{23} \mathrm{~cm}^{-2}$ along the column-density axis. Temperature profiles are almost the same, except that the intermediate zone for the $\log \xi=3$ case is $25 \%$ less than for the $\log \xi=4$ case.

As ionization or recombination timescales are specific to each ion, we analyzed the ionization structure of a whole 


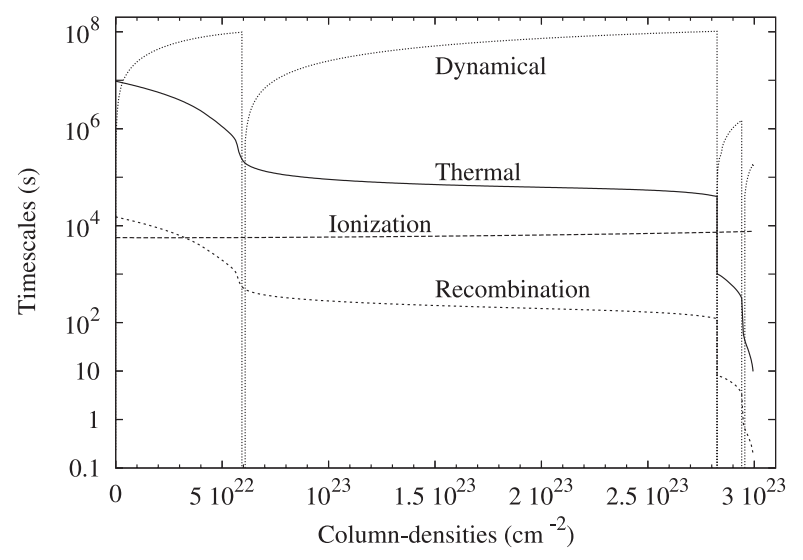

Fig. 2. The dependence of the various local timescales (s) across the cloud (as a function of the column-density): ionization timescale, recombination timescale, thermal equilibrium timescale, hydrostatic equilibrium timescale (cumulative through the slab, classical perfect gas). As ionization or recombination timescales are specific to each ion, we choose to represent FeXXVI, which represents the longest timescale (only in the hot zone for recombination, leaders are SixV, OIX, and CVII for the intermediate, outer, and end zones, respectively; see Tables 1 and A.1).

cloud (see the Appendix and Table A.1). The longest ionization timescale is equal to $8 \times 10^{3} \mathrm{~s}$ (for ion FeXXVI in the hot zone). Processes representative of the intermediate, outer, and end zones are connected with SXVI, OVIII, and CVI, respectively. The representative recombination timescale for a whole cloud is $2 \times 10^{4}$ s (again for FeXXVI in the hot zone). Representative ions for the recombination timescale in the intermediate, outer, and end zones are SiXV, OIX, and CVII, respectively.

The profiles of the timescales calculated locally across the cloud are shown in Fig. 2. We see that the photoionization timescale is roughly constant through the slab, but the recombination timescale is monotonically decreasing through the cloud since it is inversely proportional to the electron density $n_{\mathrm{e}}$ according to the scaling formula (see Eq. (3)). The actual recombination timescale calculated numerically across the cloud (see the Appendix) also depends on the temperature (not linearly) and decreases even faster. The recombination timescale is shallower if we consider various ions as representative of different zones (see Table 1).

The overall thermal timescale depends both on the temperature and the density, so it decreases with the distance from the cloud surface even more rapidly than the recombination timescale (see Table 1), and roughly follows the numerically obtained recombination timescale for a single ion. Its maximum value of $10^{7}$ at the hot surface is very high and lasts much longer than ionization or recombination timescales. We have checked that $\Lambda_{23} \sim 1$, whatever the depth for our model, including the surface layers. In other zones the thermal timescale is shorter, so most ions reach equilibrium in the timescale appropriate to the intermediate zone $\left(2 \times 10^{5} \mathrm{~s}\right)$, and we adopt this value as the longest characteristic thermal timescale of the cloud. However, we must stress that in the case of the hot zone, where highly ionized iron spectral features form, the full thermal equilibrium is hard to achieve. The temperature rises/falls slowly since both the atomic heating/cooling and the Compton heating/cooling are not very efficient. The dynamical timescale of the whole cloud obtained through integration is equal to $2 \times 10^{8} \mathrm{~s}$. Both in the hot and in the intermediate zones these timescales, when calculated separately, are long (see Table A.1). In Fig. 2 we plot the cumulative timescales for each of the zones separately, starting from the beginning of a zone.

\subsection{Cloud states and conditions for the self-consistency of the cloud response description}

The X-ray flux of AGN nuclei is strongly variable. Since the timescales corresponding to ionization/recombination equilibrium and to the dynamical equilibrium differ by a few orders of magnitude, a cloud is not always likely to achieve complete equilibrium. We now analyze the conditions of complete, or partial, equilibrium.

For this purpose, we must compare the cloud's internal timescales with the timescales of the intrinsic variability of the X-ray incident flux. AGN do not have any specific timescales but the power spectrum in Seyfert galaxies is represented well by a power law with the two breaks (e.g. Markowitz et al. 2003). Most of the power is between the high and the low frequency breaks, which depend on the central black hole mass. We can consider these frequency breaks of the power spectrum in an AGN as a characteristic range of timescales. We adopt $t_{\mathrm{X}}^{\text {short }}$ and $t_{\mathrm{X}}^{\text {long }}$ as the shortest and longest timescales of variation, respectively. With a black hole mass of $10^{7} M_{\odot}$, the appropriate timescales for regular Seyfert 1 galaxies are $t_{\mathrm{X}}^{\text {short }}=6 \times 10^{5} \mathrm{~s}$ and $t_{\mathrm{X}}^{\text {long }}=6 \times 10^{7} \mathrm{~s}$ (Markowitz et al. 2003; Papadakis 2004), and the short timescale is shorter by a factor 20 for narrow-line Seyfert 1 galaxies. For illustrative purposes, we further adopt $t_{\mathrm{X}}^{\text {short }}=10^{5} \mathrm{~s}$ and $t_{\mathrm{X}}^{\text {long }}=10^{7} \mathrm{~s}$.

The full thermal and pressure equilibrium within the cloud is achieved if the light crossing time, the longest of the ionization, recombination, or thermal timescales, and the dynamical time of the cloud are much shorter than the shortest X-ray variability timescale, i.e. $\max \left(t_{\text {light }}, t_{\text {th }}, t_{\text {ion }}, t_{\text {recomb }}, t_{\text {dyn }}\right) \ll t_{\mathrm{X}}$. On the other hand, the thermal equilibrium solution with an unchanged density profile requires $\max \left(t_{\text {light }}, t_{\text {th }}, t_{\text {ion }}, t_{\text {recomb }}\right) \ll t_{\mathrm{X}} \ll t_{\text {dyn }}$.

The second set of conditions is roughly fulfilled in our reference cloud. The ionization and recombination timescales are short for all important transitions. The thermal timescale in the intermediate zone is comparable to $t_{\mathrm{X}}^{\text {short }}$, and only the thermal timescale in the hot zone is significantly longer, which may affect the hydrogen-like iron absorption feature. The shortest dynamical timescale in the last zone is still longer than $t_{\mathrm{X}}^{\text {short }}$, so the model is appropriate to a short timescale variability. Longer systematic trends, like those in $t_{\mathrm{X}}^{\text {long }}=10^{7} \mathrm{~s}$, should be followed by computing new pressure equilibrium at the back side of the cloud, in the outer and end zones, but retaining the old density profile in the hot and intermediate zone. We postpone the computations of these hybrid models to the future.

Summarizing, our new equilibrium is fully self-consistent with an increase in the X-ray flux lasting up to $t_{X}=3 \times 10^{5} \mathrm{~s}$ (i.e. the shortest dynamical timescale, see Table 1) if the density profile is left unchanged. Results obtained for longer timescales can only serve as indicators of the trends.

\subsection{Response of the cloud structure to the change in irradiation flux}

We therefore compute a set of new cloud equilibrium solutions by increasing the incident flux and preserving the density profile from the pressure equilibrium state described in Sect. 3.1. Figure 3 shows the temperature profiles of the cloud for which the increase of flux is by a factor 3,10, and 100. Naturally, 


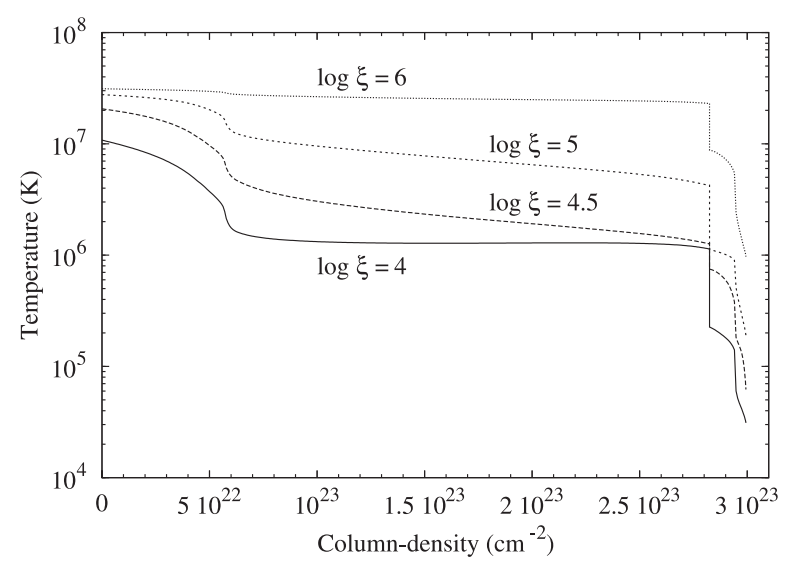

Fig. 3. The temperature profile of the cloud at the initial state $\xi=$ $10^{4} \mathrm{erg} \mathrm{cm} \mathrm{s}^{-1}$ (i.e. $\log \xi=4$ ) and after an increase of the irradiation to $\log \xi=4.5,5$ and 6 , but before a new pressure equilibrium is reached.

an increase in the X-ray flux results in a higher surface temperature. The depth of the first, hottest zone does not change, but the temperature of the intermediate zone rises. An increase in X-ray flux by a factor 100 removes the intermediate zone completely. The last two colder zones always exist, although the temperature of the back side of the cloud rises up to $10^{6} \mathrm{~K}$ for the highest irradiation flux. However, the rise in the incident flux by a factor of 3 already brings up the temperature of the outer zone to almost close to the temperature of the intermediate zone.

\subsection{Response of the cloud velocity field to the change in irradiation flux}

After the change in the incident flux, the cloud is not in pressure equilibrium any more. Pressure gradients appear that lead to local acceleration of the gas within the cloud and subsequent expansion. Figure 4 shows the gaseous, radiative, and total pressure for the initial state $\log \xi=4$ and the final state $\log \xi=4.5$. The most prominent feature is the huge positive pressure gradient at the transition between the intermediate and outer zones. Therefore, a shock may form at this location as the material strain to recover the dynamical equilibrium. It is not a classical ionization front since the whole cloud is in a new ionization equi-

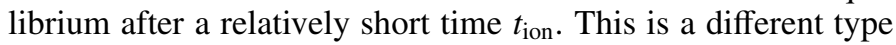
of shock that forms due to the presence of the density gradient in the original model, previously supported by the appropriate temperature gradient. This support is removed by the sudden rise in the temperature on the cold side. Since the discontinuity in the total pressure is not very large (a factor of 1.25 in case of the flux rise by a factor 3, see Fig. 4), the expected Mach number, $\mathcal{M}$, of an isothermal shock is low $\left(\mathcal{M}^{2}=1.25\right)$, a relatively weak shock forms and propagates with a speed close to the speed of sound $\mathcal{M} c_{\mathrm{s}} \sim 120 \mathrm{~km} \mathrm{~s}^{-1}$ in this zone, as we only have to consider the highest temperature $T \sim 7 \times 10^{5} \mathrm{~K}$ ahead of the thermal instability zone (see Fig. 3). Subsonic motions due to the lack of pressure balance will also appear in other parts of the cloud.

Due to the very different values of the velocity in our cloud, we thus expect that lines forming in these transition zones may have a very complex profile: part of the line may be narrow, and part of the line profile may be broad. The presence of the significant velocity gradient means that the assumption of a constant turbulence across the cloud may not be justified, but the computations of the radiative transfer with variable velocity field is beyond the scope of the present paper.

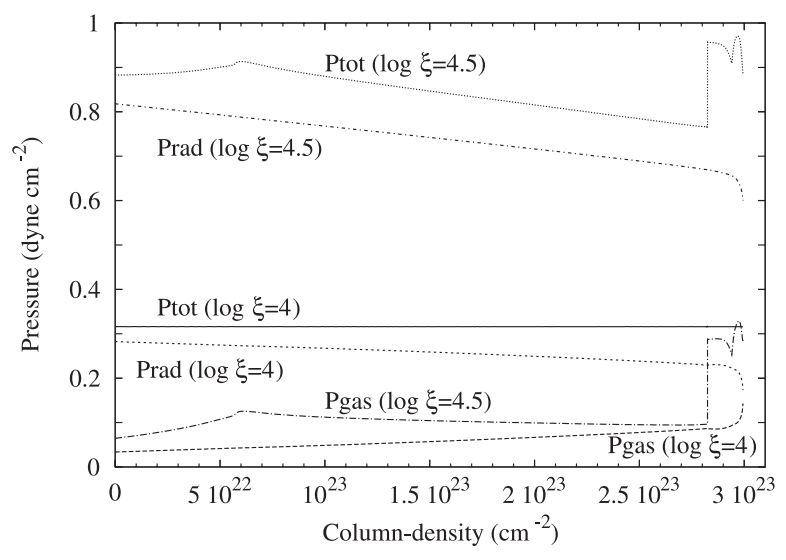

Fig. 4. Variation with the column-density of gaseous (Pgas), radiation (Prad), and total pressure (Ptot) for the $\log \xi=4$ constant total pressure case, and under illumination $\log \xi=4.5$ for a fixed density profile.

\subsection{Clouds at various distances from the nucleus}

The choice of $n_{\mathrm{o}}=10^{7} \mathrm{~cm}^{-3}$ and $\xi=10^{4}$ corresponds to the choice of the distance for a specific choice of the bolometric luminosity $L$ of the nuclear source. The domain of observed luminosities range from $10^{41} \mathrm{erg} \mathrm{s}^{-1}$ (e.g., NGC 4051) up to $10^{47} \mathrm{erg} \mathrm{s}^{-1}$ (e.g., quasars). We adopt $L=10^{45} \mathrm{erg} \mathrm{s}^{-1} \mathrm{~cm}^{-2}$ in the next calculations: the cloud is located at a distance of $10^{17} \mathrm{~cm}$ from the nucleus. All those results scale as $L / R^{2}$, and any value of $L$ can be chosen.

The results obtained for a single cloud can be generalized to a family of clouds located at various distances from the nucleus adopting scaling laws based on the very weak dependence of the results for the radiative transfer on the adopted value of the number density at the cloud surface, $n_{\mathrm{o}}$ (see Sect. 2).

We checked the underlying assumption by calculating a set of clouds for $\xi=10^{4} \mathrm{erg} \mathrm{cm} \mathrm{s}-1, N_{\mathrm{H}}=3 \times 10^{23} \mathrm{~cm}^{-2}$ and with values of $n_{\mathrm{o}}$ in the range from $10^{5}$ to $10^{11} \mathrm{~cm}^{-3}$. The temperature profiles measured as functions of the optical depth were almost identical. The density profiles as functions of the optical depth had very similar shapes and only differed in the normalization. The ratio of the density at the outer edge of the cloud to $n_{\mathrm{o}}$ was almost constant, only slowly decreasing from $1.5 \times 10^{3}$ to $10^{3}$ with the increasing density.

Therefore, as the basis of the numerical results for a single cloud, we now consider a family of clouds for $\xi=$ $10^{4} \mathrm{erg} \mathrm{cm} \mathrm{s}^{-1}, N_{\mathrm{H}}=3 \times 10^{23} \mathrm{~cm}^{-2}$, and the bolometric luminosity of the nucleus $L=10^{45} \mathrm{erg} \mathrm{s}^{-1} \mathrm{~cm}^{-2}$. The condition of constant $\xi$ in this case implies that the number density at the cloud's illuminated surface is $n_{\mathrm{o}}(R) / R_{17}^{-2}=10^{7} \mathrm{~cm}^{-3}$, where $R_{17}$ is the distance in units of $10^{17} \mathrm{~cm}$ and is higher by a factor of $10^{3}$ at the dark surface. Other quantities defined in Sect. 2 become $H(R) / R_{17}^{2}=5 \times 10^{15} \mathrm{~cm}, t_{\text {light }}(R) / R_{17}^{2}=1.7 \times 10^{5} \mathrm{~s}$, $t_{\text {ion }}(R) / R_{17}^{2}=8.0 \times 10^{3}-30 \mathrm{~s}, t_{\mathrm{rec}}(R) / R_{17}^{2}=2 \times 10^{4}-20 \mathrm{~s}$, $t_{\mathrm{th}}(R) / R_{17}^{2}=2 \times 10^{5}-60 \mathrm{~s}$, and $t_{\mathrm{dyn}}(R) / R_{17}^{2}=10^{8}-4 \times 10^{5} \mathrm{~s}$.

Using these expressions and a variability timescale of the source between $10^{5} \mathrm{~s}$ and $10^{7} \mathrm{~s}$, we can estimate the kind of equilibrium reached by our medium as a function of the distance. The results are shown in Fig. 5, where several regions can be defined as a function of the distance of our cloud from the nucleus. Note that those results only depend on the value of the ionization parameter, $\xi$, and the cloud column density, $N_{\mathrm{H}}$, and this figure can be scaled with various values of the luminosity $L$. We 


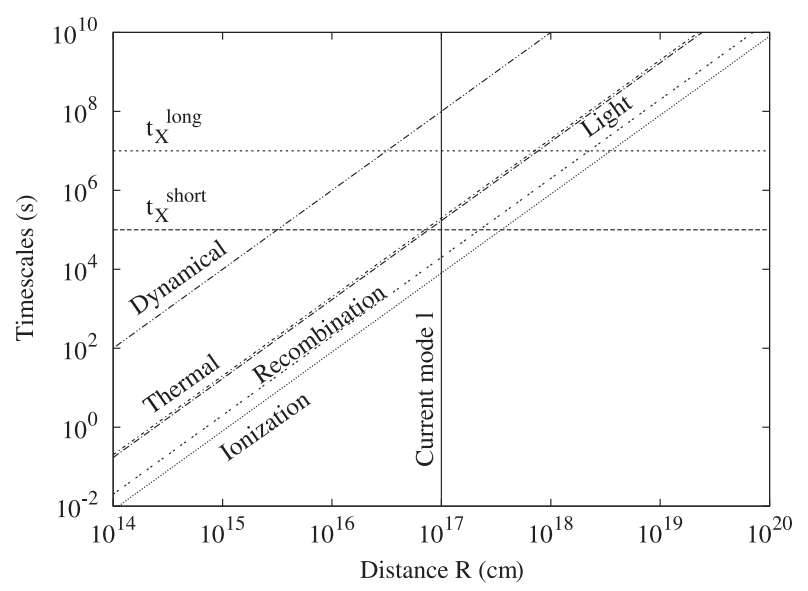

Fig. 5. The dependence of the maximum value within the cloud of various timescales as a function of the distance from the nucleus for a source with luminosity $L=10^{45} \mathrm{erg} \mathrm{s}^{-1}$. We represent the bounds of the characteristic range of variability of the source, i.e. the the shortest timescale $t_{\mathrm{X}}^{\text {short }}$ and longest timescale $t_{\mathrm{X}}^{\text {long }}$, light travel time, ionization timescale, recombination timescale, thermal timescale, and dynamical timescale. This value of the thermal timescale excludes the hot zone, where it is up to 50 times longer (see Table 1). A vertical line indicates the current model presented here (i.e. hydrogen surface density $n_{\mathrm{o}}=10^{7} \mathrm{~cm}^{-3}$ which is equivalent to $\left.R=10^{17} \mathrm{~cm}\right)$.

see from Fig. 5 that in general we can have four basic types of clouds:

- full (dynamical + thermal) equilibrium, $R<3 \times 10^{16} \mathrm{~cm}$;

- thermal equilibrium, $3 \times 10^{16} \mathrm{~cm}<R<3 \times 10^{18} \mathrm{~cm}$;

- no ionization equilibrium, $3 \times 10^{17} \mathrm{~cm}<R<5 \times 10^{18} \mathrm{~cm}$;

- clouds at $R>5 \times 10^{18} \mathrm{~cm}$.

The innermost clouds should be described using full equilibrium computations. The second group of clouds shows the departure from the dynamical equilibrium, but their ionization equilibrium is preserved. Their ionization state should be roughly correlated with the X-ray flux measured at any given moment, and they can be represented as described in Sect. 3.3. In particular, a shock forms and propagates at velocity $\sim 120 \mathrm{~km} \mathrm{~s}^{-1}$, whatever the distance $R$, as the variability timescale $t_{\mathrm{X}}$ and the extent of the outer zone are both higher than the time and displacement, respectively, of the matter when the sound speed is reached (see Sect. 3.4). Clouds farther up are so extended that separate parts of the cloud respond to different continua, as their light travel time is comparable to the timescales of the X-ray source variability. Only complete time-dependent computations of the radiative transfer, with the adopted light-curve, are appropriate to analyzing such clouds, and at present we are unable to perform such computations. For the last group of very distant clouds, the cloud size is so extended that the assumption of a single cloud breaks down and we are dealing with an extended continuous wind.

\section{Discussion}

In this paper we have considered the complexity of the response of the warm absorber to the variable X-ray incident flux. To assess the cloud response, we mostly concentrated on comparing two states of a single warm absorber cloud: an initial irradiated cloud at pressure equilibrium and a cloud suddenly irradiated by a three times higher flux; i.e. that cloud could reach new thermal and ionization equilibrium under enhanced irradiation, but the time was too short to reach a new pressure equilibrium. The model can be used with the presently available and future observational data.

\subsection{Observational applications}

Below we give a few exemplary applications of our representative cloud results, but we are not yet focused on fitting a specific object. More precise modeling should use a family of solutions.

\subsubsection{Soft X-ray variability}

Enhanced irradiation leads to higher ionization of the cloud and lower opacity, particularly in the soft X-ray band. Indeed, observations based on RXTE monitoring show enhancement of the energy-dependent fractional variability amplitude below $5 \mathrm{keV}$ (Markowitz et al. 2003). The best-studied case of a Seyfert 1 galaxy MCG-6-30-15 shows enhanced variability in the $0.2-4 \mathrm{keV}$ band approximately equal to $30 \%$ (Fabian et al. 2002; Ponti et al. 2004). The variability below $0.2 \mathrm{keV}$ and above $5 \mathrm{keV}$ must be intrinsic, as it reflect the changes in the level (and possibly the slope) of the continuum, but the excess $0.2-4 \mathrm{keV}$ may be due to the opacity changes in the warm absorber (e.g. Sako et al. 2002). This effect has been studied for this object using constant density clouds (Gierlinski \& Done 2006).

Using our density-stratified clouds, we calculated the ratio of the two spectra (models with $\log \xi=4$ and $\log \xi=4.5$ ). Since the change in the flux by a factor 3 is rather extreme as compared to the observed variability in MCG-6-30-15, we only consider the model with $\log \xi=4.1$ (i.e. increase of $\sim 26 \%$ in the incident flux) without adjustment of the pressure and another one with $\log \xi=4.1$ in constant pressure equilibrium. In Fig. 6 we show the ratios of the two spectra plotted with the usual resolution from the computation of the fractional variability amplitude. We also show the fractional variability amplitude calculated from one of the data sets obtained for MCG-6-30-15 (XMM observation in 2000, rev. 303; see Goosmann et al. 2006). Indeed, there is a strong enhancement of the variability at $1-2 \mathrm{keV}$. The maximum value of $\sim 50 \%$ of the fractional variability for the $\log \xi=4.1$ case is close to the MCG-6-30-15 case. This suggests that the variable ionization of the warm absorber is a promising contributor to the overall variability. If so, such enhanced variability in the soft X-ray band peaking at $1 \mathrm{keV}$ should be seen only in the sources that show the presence of the warm absorber. Our model shows slightly too much absorption for this data set, and a better fit would be reached with a lower column-density for the same value of $\xi$. It is also interesting to point out that the fractional variability enhancement obtained without the pressure equilibrium extends more into high energies and seems to fit the data better. This would suggest the warm absorber distance larger than $3 \times 10^{15} \mathrm{~cm}$ since the medium had no time to reach dynamical equilibrium (see Sect. 3.5 ).

\subsubsection{Observational determination of the column density}

An interesting trend was found toward an increasing equivalent column density of the absorber with an increase in the ionization parameter for the species (NGC 5548, Steenbrugge et al. 2005; NGC 4051, Ogle et al. 2004; NGC 7469, Scott et al. 2005). It is therefore interesting to see whether such a trend is explained by our constant-pressure clouds. Therefore, we selected the ions studied by Scott et al. (2005) and performed a similar analysis, i.e. we used the ion column densities from our simulations (for the initial cloud and the cloud with increased irradiation, out of 


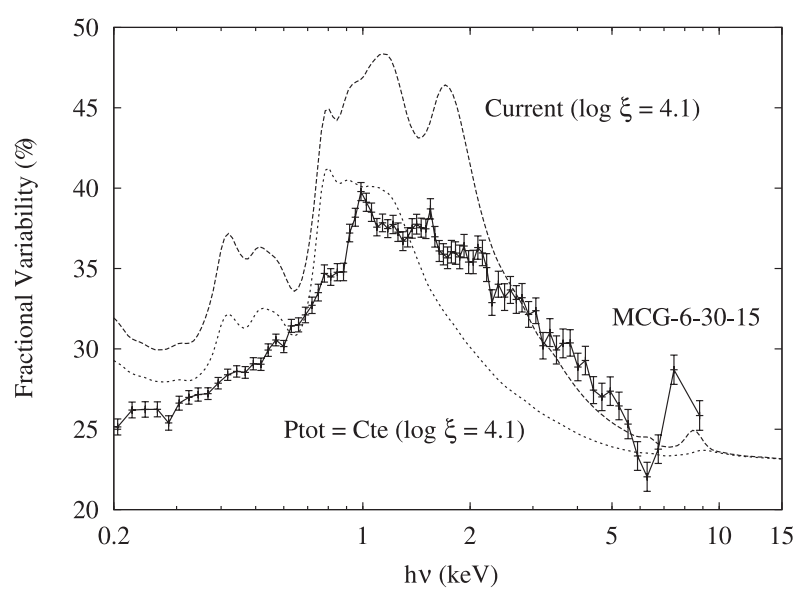

Fig. 6. The fractional variability amplitude (here in $\%$, defined as $\left.2\left|F_{1}-F_{2}\right| /\left(F_{1}+F_{2}\right)\right)$ calculated using the $\log \xi=4$ spectrum as a reference for two models of increasing flux to $\log \xi=4.1$, the first one as described in this study (Current) and the second one in total pressure equilibrium (Ptot $=\mathrm{Cte})$. Those quantities are shown for a wide energy range: 0.2 to $15 \mathrm{keV}$. Some difference is seen in the width of the horizontal zone between 1 and $2 \mathrm{keV}$, and for the features around $7 \mathrm{keV}$ (iron $K \alpha$ line region). The spectral resolution is 10 (Gaussian shape). The overall level of the amplitude reflects the assumption about the increase in the flux, but the energy-dependent features can be compared to the features in the observed fractional variability amplitudes. The exemplary data points represent a $100 \mathrm{ks}$ XMM observation of MCG-6-30-15 (Goosmann et al. 2006).

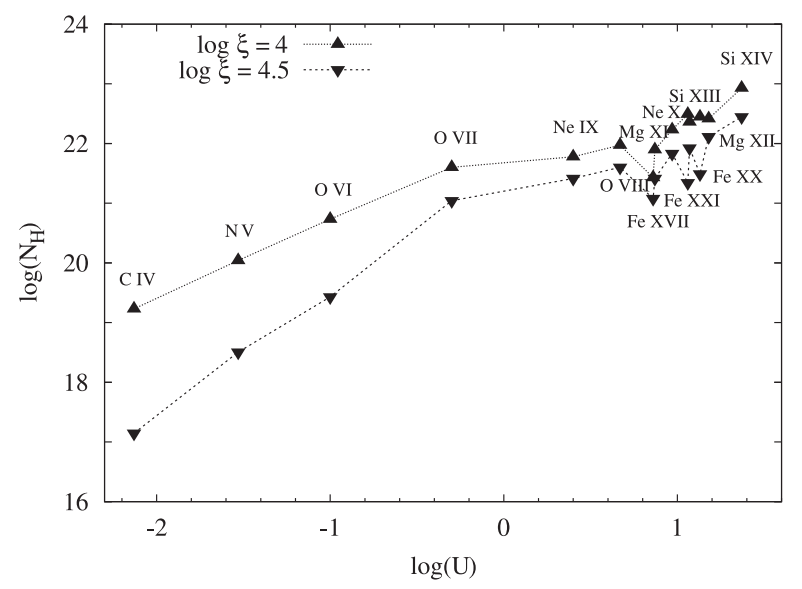

Fig. 7. Equivalent hydrogen column density vs. ionization parameter for some ions selected in our reference cloud in its initial state $(\log \xi=4)$ and with an increased illumination ( $\log \xi=4.5)$. The apparent systematic increase reproduces the trend seen in the data of Scott et al. (2005).

pressure equilibrium) and inverted them to equivalent hydrogen column using only abundance values. The results are shown in Fig. 7. We see that the trend seen in the data is explained by our model in a natural way. The ratio of almost five orders of magnitude between the results for FeXX, MgXII, SiXII, SiXIV, and CIV is explained well by the model out of dynamical equilibrium. On the other hand, our column densities are lower than those obtained by Scott et al. (2005). Extensive modeling with the use of other cloud parameters will be necessary to see whether the specific values characteristic of NGC 7469 can be accommodated within our model.
Table 2. Kinematical widths of the X-ray absorption lines estimated from our cloud model in comparison with the line widths measured by Scott et al. (2005). The third column gives the dominant location of the ion (I, intermediate zone; O, outer zone).

\begin{tabular}{cllcc}
\hline $\begin{array}{c}\text { Wavelength } \\
{[\mathrm{A}]}\end{array}$ & Abs. line & Zone & $\begin{array}{c}\text { Model } \\
{\left[\mathrm{km} \mathrm{s}^{-1}\right]}\end{array}$ & $\begin{array}{c}\text { Measured } \\
{\left[\mathrm{km} \mathrm{s}^{-1}\right]}\end{array}$ \\
\hline 6.18 & SiXIV & I & 150 & $620 \pm 690$ \\
6.65 & SiXIII & I & 150 & $1350 \pm 900$ \\
8.42 & MgXII & I & 150 & $500 \pm 160$ \\
9.17 & MgXI & I \& O & 150 & $680 \pm 410$ \\
11.55 & NeIX & O & 60 & $1020 \pm 520$ \\
12.13 & NeX & I \& O & 150 & $430 \pm 270$ \\
12.28 & FeXXI & I & 150 & $890 \pm 570$ \\
12.82 & FeXX & I & 150 & $1070 \pm 340$ \\
13.45 & NeIX & O & 60 & $1050 \pm 490$ \\
15.01 & FeXVII & O & 60 & $650 \pm 320$ \\
\hline
\end{tabular}

\subsubsection{Line widths}

The predicted line widths depend on the cloud location for a fixed nuclear source properties. Clouds located closer in reach equilibrium much faster and the velocity gradient is smaller. Clouds located much farther away will also show smaller velocity gradients since they are too extended to react to the variable radiation flux. Therefore, the measurement of the line width will provide an independent limit to the cloud distance and an additional test of the constant pressure cloud models.

We illustrate this issue in Table 2. We take the measured kinematical width of absorption lines measured in the X-ray spectrum of NGC 7469 by Scott et al. (2005) and compare them with our estimates based on the location of the ions (i.e. the sound speed velocity of the zone). As a result, lines identified by Scott et al. (2005) all come either from the intermediate or from the outer zone in our cloud. Measured widths in the X-ray band are always larger and are given with significant errors. It is probable that many lines are actually blends, and the resolution in X-ray spectra is not yet satisfactory for testing the model. Our model predicts many more strong absorption lines in this energy band. Lines are measured much more precisely in the UV part of the spectrum, so in the future our study will be extended into the UV spectral region. The available measurements for NGC 7469 UV data cannot be used immediately for our purpose since line intensities and shifts were measured for the line widths fixed at the same value for all narrow absorption components $\left(61 \mathrm{~km} \mathrm{~s}^{-1}\right.$ and $45 \mathrm{~km} \mathrm{~s}^{-1}$ for two kinematical components; Scott et al. 2005).

\subsection{Further improvements in modeling}

Our approach to modeling the time-dependent response of the cloud to irradiation was a first step in this direction. It is well known that the problem is complicated, and the best-studied classical example is the time-dependent evolution of the expanding $\mathrm{H}^{+}$region around a newly born star (see Osterbrock 1974). Unfortunately, our situation is more complex since we do not deal with a single ionization front, and simple analytical estimates do not apply very easily to our case. However, further improvement of the description of the cloud dynamics is necessary.

A particularly important and difficult problem appears if clouds are at large distances from the center. Blustin et al. (2005) argue that the warm absorber is located in most sources at the distance $\sim 1 \mathrm{pc}$. Our cloud at such a distance is far from radiative equilibrium. In this case time-dependent radiation transfer computations seem to be necessary. 
Table A.1. Table of the column densities (c, in $\mathrm{cm}^{-2}$ ), ionization (i) and recombination (r) timescales (in s) for all 4 zones in the medium for the representative, i.e. comparable column density for both ionization states, ions of $\mathrm{Fe}, \mathrm{S}$ and $\mathrm{Si}, \mathrm{O}$, and $\mathrm{C}$ in the hot, intermediate, outer, and end zones, respectively.

\begin{tabular}{lccccc}
\hline \hline Property & Hot & Interm. & Outer & End & All \\
\hline FeXXV c & $5 \times 10^{17}$ & $10^{18}$ & $2 \times 10^{10}$ & 2 & $2 \times 10^{18}$ \\
FeXXVI c & $7 \times 10^{17}$ & $10^{17}$ & $4 \times 10^{7}$ & $3 \times 10^{-4}$ & $9 \times 10^{17}$ \\
FeXXVII c & $6 \times 10^{17}$ & $5 \times 10^{15}$ & $3 \times 10^{4}$ & $2 \times 10^{-8}$ & $7 \times 10^{17}$ \\
FeXXVI r & $2 \times 10^{4}$ & 500 & 8 & 0.8 & $2 \times 10^{4}$ \\
FeXXVII r & $10^{4}$ & 400 & 7 & 0.7 & $10^{4}$ \\
\hline SXVI c & $8 \times 10^{16}$ & $2 \times 10^{18}$ & $10^{15}$ & $2 \times 10^{10}$ & $2 \times 10^{18}$ \\
SXVII c & $9 \times 10^{17}$ & $10^{18}$ & $2 \times 10^{13}$ & $2 \times 10^{7}$ & $2 \times 10^{18}$ \\
SXVI i & 800 & $10^{3}$ & $10^{3}$ & $10^{3}$ & $10^{3}$ \\
\hline SiXIV c & $8 \times 10^{16}$ & $3 \times 10^{18}$ & $2 \times 10^{16}$ & $2 \times 10^{12}$ & $3 \times 10^{18}$ \\
SiXV c & $2 \times 10^{18}$ & $4 \times 10^{18}$ & $5 \times 10^{14}$ & $5 \times 10^{9}$ & $6 \times 10^{18}$ \\
SiXV r & $5 \times 10^{4}$ & $2 \times 10^{3}$ & 30 & 3 & $5 \times 10^{4}$ \\
\hline OVIII c & $3 \times 10^{16}$ & $2 \times 10^{18}$ & $3 \times 10^{18}$ & $2 \times 10^{17}$ & $7 \times 10^{18}$ \\
OIX c & $4 \times 10^{19}$ & $10^{20}$ & $3 \times 10^{18}$ & $7 \times 10^{15}$ & $2 \times 10^{20}$ \\
OVIII i & 40 & 50 & 100 & 200 & 200 \\
OIX r & $2 \times 10^{5}$ & $7 \times 10^{3}$ & 100 & 10 & $2 \times 10^{5}$ \\
\hline CVI c & $2 \times 10^{15}$ & $10^{17}$ & $3 \times 10^{17}$ & $6 \times 10^{17}$ & $10^{18}$ \\
CVII c & $2 \times 10^{19}$ & $7 \times 10^{19}$ & $3 \times 10^{18}$ & $2 \times 10^{17}$ & $10^{20}$ \\
CVI i & 10 & 10 & 10 & 30 & 30 \\
CVII r & $6 \times 10^{5}$ & $10^{4}$ & 200 & 20 & $6 \times 10^{5}$ \\
\hline & & & & &
\end{tabular}

Acknowledgements. We thank S. Collin, C. Done, T. Kallman, F. Nicastro, and I. Shlosman for helpful discussions. We also thank A.-M. Dumont for providing some help with the TITAN code. A.C. Gonçalves acknowledges support from the Fundação para a Ciência e a Tecnologia, Portugal, under grant no. BPD/11641/2002. Part of this work was supported by grant 1P03D00829 of the Polish State Committee for Scientific Research, and the Laboratoire Européen Associé Astrophysique Pologne-France.

\section{Appendix}

In order to obtain the ionization timescale $t_{\text {ion }}$ of a whole cloud, we calculate these timescales for all ions $i$ separately, as local quantities within a cloud,

$\left(t_{\text {ion }}^{i}\right)^{-1}=4 \pi \int_{\chi^{i} / h}^{+\infty} \frac{J_{v}}{h v} \sigma^{i}(v) \mathrm{d} v$,

where $\sigma^{i}$ is the ionization cross-section and $\chi^{i}$ the ionization energy of a given ion. Equation (2) is derived analytically by assuming a normal flux, neglecting the reflected flux (it is less than $10 \%$ the incident flux), and using a classical formula for the cross-section (Osterbrock 1974). The recombination timescale for each ion is estimated from the semi-analytical formulae of Verner \& Ferland (1996)

$\left(t_{\mathrm{rec}}^{i}\right)^{-1}=n_{\mathrm{e}}\left(\alpha_{r}(T)+\alpha_{\text {diel }}(T)\right)$,

where $\alpha_{\mathrm{r}}(T)$ is the radiative recombination coefficient and $\alpha_{\text {diel }}(T)$ the coefficient of the dielectronic recombination. Induced radiative recombination is negligible, hence this timescale does not depend on the flux.

As ionization or recombination timescales are specific to each ion, we analyzed the ionization structure of a whole cloud. An illustration of the method is shown in Table A.1, where we give the column densities, ionization, and recombination timescales, zone per zone, for FeXXV-XXVII, SXVI-XVII,
SiXIV-XV, OVIII-IX, and CVI-VII, which are relevant for our model. Recombination timescales for fully ionized elements are quite long (up to $2 \times 10^{8}$ s for hydrogen), but hydrogen and helium are almost completely ionized, and since these ions do not undergo frequent transitions, we determine the recombination timescale in each zone by identifying the dominant processes (i.e. both ions in interaction with this process must have roughly the same abundances). Although in Table A. 1 the longest recombination timescale is $6 \times 10^{5} \mathrm{~s}$ for the carbon in the hottest zone, such transitions are of marginal importance. Therefore, the representative recombination timescale for a whole cloud is $2 \times$ $10^{4} \mathrm{~s}$ (again for FeXXVI in the hot zone). Representative ions for the recombination timescale in the intermediate, outer, and end zones are SiXIV, OIX, and CVII, respectively.

Since these ionization and recombination timescales depend, respectively, on the incident flux and the density $(F=8 \times$ $10^{9} \mathrm{erg} \mathrm{s}^{-1} \mathrm{~cm}^{-2}$ and $n_{\mathrm{o}}=10^{7} \mathrm{~cm}^{-3}$ in our reference cloud), we generalize the obtained values to Eqs. (2) and (3).

\section{References}

Allen, C. W. 1973 (London: University of London, Athlone Press), 3rd edn. Ashton, C. E., Page, M. J., Branduardi-Raymont, G., \& Blustin, A. J. 2006, MNRAS, 366, 521

Bottorff, M. C., Korista, K. T., \& Shlosman, I. 2000, ApJ, 537, 134

Blustin, A. J., Page, M. J., Fuerst, S. V., Branduardi-Raymont, G., \& Ashton, C. E. 2005, A\&A, 431, 111

Chelouche, D., \& Netzer, H. 2005, ApJ, 625, 95

Collinge, M. J., Brandt, W. N., Kaspi, S., et al. 2001, ApJ, 557, 2

Chevallier, L., Collin, S., Dumont, A.-M., et al. 2006, A\&A, 449, 493

Dumont, A.-M., Abrassart, A., \& Collin, S. 2000, A\&A, 357, 823

Dumont, A.-M., Czerny, B., Collin, S., \& Życki, P. T. 2002, A\&A, 387, 63

Dumont, A.-M., Collin, S., Paletou, F., et al. 2003, A\&A, 407, 13

Elvis, M. 2000, ApJ, 545, 63

Fabian, A. C., Vaughan, S., Nandra, K., et al. 2002, MNRAS, 335, L1

Gabel, J. R., Crenshaw, D. M., Kraemer, S. B., et al. 2003, ApJ, 595, 120

Gierliński, M., \& Done, C. 2006, MNRAS, L64

Gonçalves, A. C., Collin, S., Dumont, A.-M., et al. 2006, A\&A, 451, L23

Goosmann, R. W., Czerny, B., Mouchet, M. et al. 2006, A\&A, 454, 741

Kaastra, J. S., Steenbrugge, K. C., Raassen, A. J. J., et al. 2002, A\&A, 386, 427

Kaspi, S., Brandt, W. N., George, I. M., et al. 2002, ApJ, 574, 643

Krolik, J. H., \& Kriss, G. A. 1995, ApJ, 447, 512

Krolik, J. H., McKee, C. F., \& Tarter, C. B. 1981, ApJ, 249, 422

Krongold, Y., Nicastro, F., Brickhouse, N. S., et al. 2003, ApJ, 597, 832

Markowitz, A., Edelson, R., Vaughan, S., et al. 2003, ApJ, 593, 96

Murray, N., \& Chiang, J. 1995, ApJ, 454, L105

Murray, N., \& Chiang, J. 1997, ApJ, 474, 91

Murray, N., \& Chiang, J. 1998, ApJ, 494, 125

Netzer, H. 1993, ApJ, 411, 594

Netzer, H. 1996, ApJ, 473, 781

Netzer, H., Kaspi, S., Behar, E., et al. 2003, ApJ, 599, 933

Ogle, P. M., Mason, K. O., Page, M. J., et al. 2004, ApJ, 606, 151

Osterbrock, D. E. 1974, Astrophysics of Gaseous Nebulae (San Francisco: W.H. Freeman and Company)

Papadakis, I. E. 2004, MNRAS, 348, 207

Piconcelli, E., Jimenez-Bailón, E., Guainazzi, M., et al. 2004, MNRAS, 351, 161

Pietrini, P., \& Torricelli-Ciamponi, G. 2000, A\&A, 363, 455

Ponti, G., Cappi, M., Dadina, M., \& Malaguti, G. 2004, A\&A, 417, 451

Proga, D., \& Kallman, T. R. 2004, ApJ, 616, 688

Proga, D., Stone, J. M., \& Kallman, T. R. 2000, ApJ, 543, 686

Różańska, A., Goosmann, R., Dumont, A.-M., \& Czerny, B. 2006, A\&A, 452, 1 Rybicki, G. B., \& Hummer, D. G. 1991, A\&A, 245, 171

Sako, M., et al. 2002, X-ray Spectroscopy of AGN with Chandra and XMM-Newton, 191

Scott, J. E., Kriss, G. A., Lee, J. C., et al. 2005, ApJ, 634, 193

Smith, M. D., \& Raine, D. J. 1988, MNRAS, 234, 297

Steenbrugge, K. C., Kaastra, J. S., Crenshaw, D. M., et al. 2005, A\&A, 434, 569

Torricelli-Ciamponi, G., \& Courvoisier, T. J.-L. 1998, A\&A, 335, 881

Verner, D. A., \& Ferland, G. J. 1996, ApJS, 103, 467 